\title{
Becker, Uwe
}

\section{Alter, Armut, Schulden. Warum Prävention ein Euphemismus ist}

Haushalt in Bildung \& Forschung 4 (2015) 3, S. 71-82

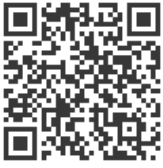

Quellenangabe/ Reference:

Becker, Uwe: Alter, Armut, Schulden. Warum Prävention ein Euphemismus ist - In: Haushalt in Bildung \& Forschung 4 (2015) 3, S. 71-82 - URN: urn:nbn:de:0111-pedocs-203832 - DOI: 10.25656/01:20383

https://nbn-resolving.org/urn:nbn:de:0111-pedocs-203832

https://doi.org/10.25656/01:20383

in Kooperation mit / in cooperation with:

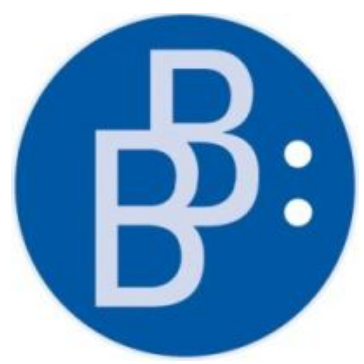

https://www.budrich.de

\section{Nutzungsbedingungen}

Gewährt wird ein nicht exklusives, nicht übertragbares, persönliches und beschränktes Recht auf Nutzung dieses Dokuments. Dieses Dokument ist ausschließlich für den persönlichen, nicht-kommerziellen Gebrauch bestimmt. Die Nutzung stellt keine Übertragung des Eigentumsrechts an diesem Dokument dar und gilt vorbehaltlich der folgenden Einschränkungen: Auf sämtlichen Kopien dieses Dokuments müssen alle Urheberrechtshinweise und sonstigen Hinweise auf gesetzlichen Schutz beibehalten werden. Sie dürfen dieses Dokument nicht in irgendeiner Weise abändern, noch dürfen Sie dieses Dokument für öffentliche oder kommerzielle Zwecke vervielfältigen, öffentlich ausstellen, aufführen, vertreiben oder anderweitig nutzen.

Mit der Verwendung dieses Dokuments erkennen Sie die Nutzungsbedingungen an.

\section{Terms of use}

We grant a non-exclusive, non-transferable, individual and limited right to using this document.

This document is solely intended for your personal, non-commercial use. Use of this document does not include any transfer of property rights and it is conditional to the following limitations: All of the copies of this documents must retain all copyright information and other information regarding legal protection. You are not allowed to alter this document in any way, to copy it for public or commercial purposes, to exhibit the document in public, to perform, distribute or otherwise use the document in public.

By using this particular document, you accept the above-stated conditions of use.

\section{Kontakt / Contact:}

\section{peDOCS}

DIPF | Leibniz-Institut für Bildungsforschung und Bildungsinformation Informationszentrum (IZ) Bildung

E-Mail:pedocs@dipf.de

Internet: www.pedocs.de

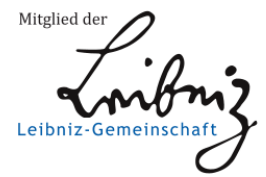




\section{Haushalt in}

Forschung

Sozioökonomische Allgemein-, Ernährungs- und Verbraucherbildung
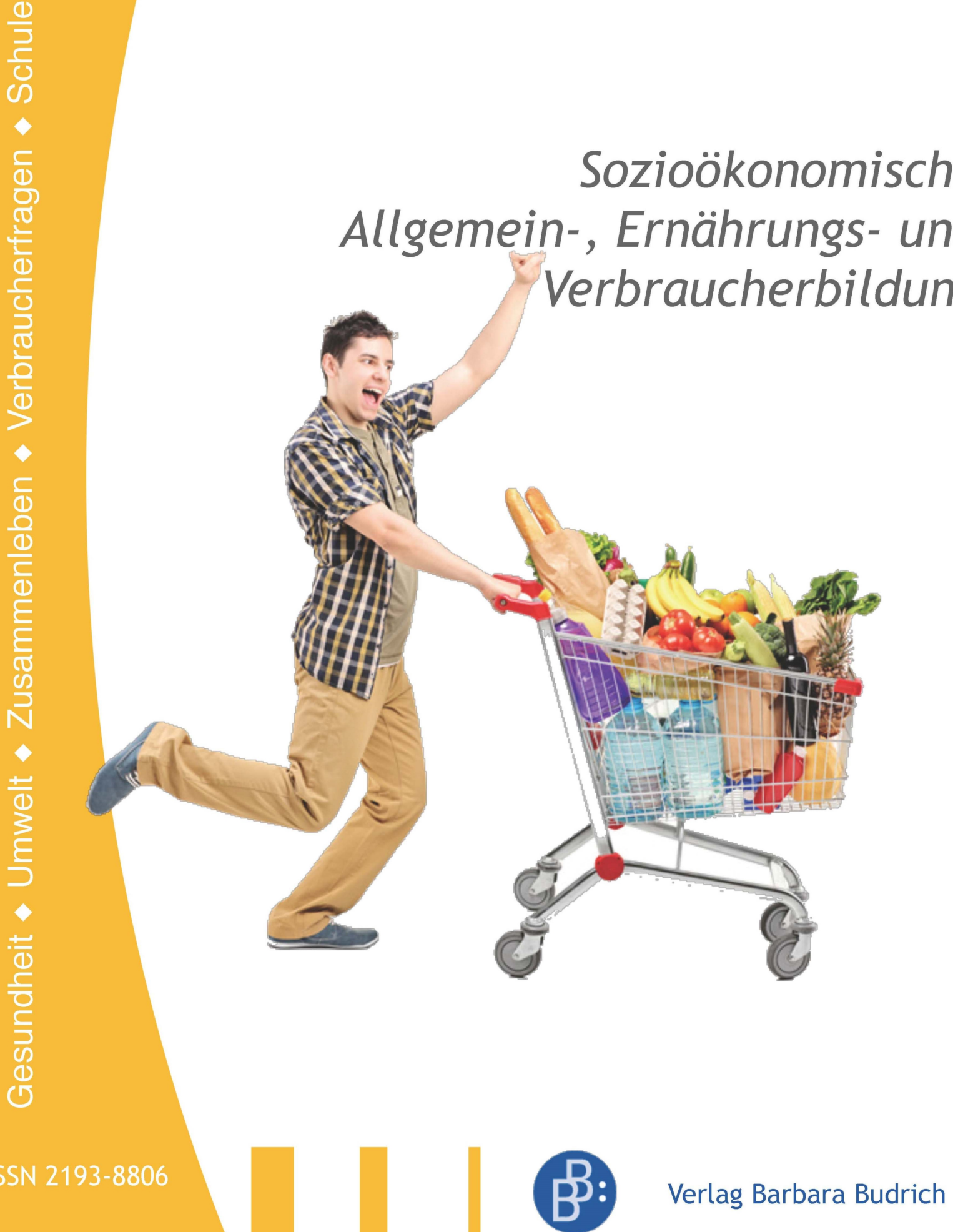
Inhaltsverzeichnis |

Werner Brandl

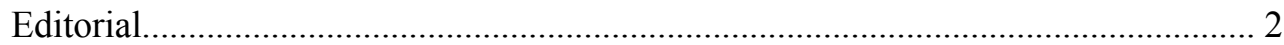

Reinhold Hedtke

Sozioökonomische Bildung................................................................................. 3

Angela Häußler

Fokus Haushalt - Überlegungen zu einer sozio-ökonomischen Fundierung der

Verbraucherbildung.

Uta Meier-Gräwe

Caring. Cooking. Cleaning. Warum wir die Arbeit des Alltags gesellschaftlich neu und geschlechtergerecht verteilen müssen.

Jana Rückert-John

Der Wandel des Ernährungsalltags als Herausforderung für die Ernährungs- und

Verbraucherkommunikation

Carolin Kölzer \& Volker Schwier

Sozioökonomische Bildung im Sachunterricht der Grundschule - mehr

als ,work, earn, play“.

Stefanie Nolte

Mit reflektierten Konsumentscheidungen die Zukunft fairändern - eine

Zukunftswerkstatt.

Uwe Becker

Alter, Armut, Schulden. Warum Prävention ein Euphemismus ist.

Werner Brandl

Reinhold Hedtke (Hrsg.). Was ist und wozu Sozioökonomie?.

Angela Häußler \& Barbara Methfessel

Uta Meier-Gräwe (Hrsg.). Die Arbeit des Alltags. Gesellschaftliche Organisation

und Umverteilung.

Anna Maria Hoff

Jana Rückert-John (Hrsg.). Soziale Innovation und Nachhaltigkeit.

Perspektiven sozialen Wandels.

HaBiFo-Fachtagung und Preis 2016. 
Warum Prävention ein Euphemismus ist

\section{Uwe Becker}

\section{Alter, Armut, Schulden. Warum Prävention ein Euphemismus ist}

Das Wort Prävention leitet sich bekanntlich von praevenire ab, wörtlich übersetzt ,zuvorkommen' oder auch ,verhüten'. Prävention bezeichnet folglich der Sache nach eine vorbeugende Maßnahme und zielt auf Vermeidung von Effekten, die kalkulatorisch ohne diese Maßnahme mit mehr oder weniger Evidenz für die Zukunft zu erwarten sind.

Schlüsselwörter: Prävention, Alter, Armut, Schulden

\section{Einleitung}

Nicht selten setzt ein Nachdenken über präventive Maßnahmen reflexiv ein, wird also erst durch den Eintritt erster negativer Effekte initiiert, um eine Verschärfung oder Perpetuierung dieser Effekte zu vermeiden. Das politische Bemühen um eine präventive Finanzkompetenz, fokussiert auf die Vermeidung einer Schulden- und Armutsentwicklung, ist ein Beispiel für diese Initiation. Der Ausgangspunkt dieser politisch-strategischen Überlegungen sind aber nicht nur Versäumnisse präventiven Handelns der Vergangenheit. Vielmehr sind politische Maßnahmen der Präventionen auch gezielt unterwandert und ihnen ist politisch absichtsvoll entgegengearbeitet worden. Anders gesagt: Die Armutsentwicklung in Deutschland ist auch Ergebnis einer politischen Programmatik, die bis heute unter Stichworten wie Schuldenbremse, Übernahme von Eigenverantwortung, Umbau des Sozialstaats, Zukunft der sozialen Sicherungssysteme oder „fordern und fördern“ ihre Alternativlosigkeit zelebriert. Diese Programmatik wird zudem mit Schlagworten wie Generationen-, Chancenoder Befähigungsgerechtigkeit eine eigentümliche pseudophilosophische Legitimation verliehen. Dem Mangel an Finanzkompetenz inklusive seiner Auswirkung auf die Armutsentwicklung in Deutschland nachzugehen, heißt quasi politische Grundlagenforschung $\mathrm{zu}$ betreiben.

\section{Prävention fängt mit Strukturanalyse an}

Prävention muss die strukturellen Ursachen der Armutsentwicklung analysieren. Andernfalls besteht die Gefahr, dass sie auf eine Art Empowerment-Strategie für finanzinkompetente Transferleistungsbezieher reduziert wird. Ein bezeichnendes 


\section{Warum Prävention ein Euphemismus ist}

Beispiel für diese Art der respektlosen Fürsorgeleistung in Sachen Armutsprävention ist wohl der Rezeptkatalog des Jobcenters in Pinneberg, der „Arbeitslosengeld II Ratgeber“ (Jobcenter Kreis Pinneberg, 2013). Hier erfährt der im ALG II-Bezug stehende Bürger in Comic-Manier zubereitet, wie er in Sachen Haushaltsführung zu sportiven Kompetenzsteigerungen befähigt werden kann. Hartz-IV-Empfänger sollten weniger Fleisch essen, auf Mineralwasser im Sechserpack zugunsten von Leitungswasser verzichten, die Möbel auf Notwendigkeit und denkbare Verkaufsstrategien hin sichten, die Heizkörper nicht zustellen, den Standby-Betrieb elektronischer Geräte ausstellen, in alle Spülkästen moderne Wasserspülungen mit Stopptaste einbauen, duschen statt Vollbad und, ganz wichtig: „Gehen Sie nie hungrig einkaufen. Denn dann landen meist mehr Lebensmittel in Ihrem Einkaufswagen, als Sie zeitnah verbrauchen können“ (Gehen Sie nie hungrig einkaufen, 2013, S. 15).

\section{Armut und Verschuldung - mehrdimensionale Ursachen}

Der Mangel eines finanziell überlegten, energiebewussten, Überfluss vermeidenden Konsums, also der Mangel an einer insgesamt haushälterisch kompetenten Lebensführung ist kein Privileg der Ober- und Mittelschicht. Sicher ist auch der ein oder andere lebenspraktische Ratschlag wie Armut effizienter privat verwaltet und gestaltet werden kann nicht sinnlos. Aber wenn auf didaktische infantile Weise das strukturelle Problem steigender Armut in Deutschland durch konsumethisches Verhaltenstraining der von Armut Betroffenen gelöst werden soll, dann ist dies nichts anderes als ein weiterer Baustein der bürokratischen Diskriminierung von Menschen in Arbeitslosigkeit. Das gegenwärtige Desaster der Verschuldung, der privaten und der öffentlichen Armut inklusive ihrer wachsenden Tendenz, ist weder auf eine Ursache allein, noch auf eine Zielgruppe reduzierbar. Die Problematik ist begründet in einer vernetzten, vielschichtigen Kausalität, und dieses Netzt gilt es zu entflechten, wenn der Ruf nach Prävention angemessene politische Forderungen und Strategien suchen will. Was die Zielgruppe anbelangt, so fallen einem durchaus ganz andere ein, die diesbezüglich einen korrigierenden Fort- und Weiterbildungsbedarf hätten. Der kalte Zynismus, mit dem irische Banker die Liquiditätskrise der Banken als Erpressungsinstrument des Staates ausgenutzt haben und ihren von Raffgier getriebenen Vorteil gesucht haben, ist bezeichnend für das Maß an Gemeinwohl schädigender Verwahrlosung von Teilen dieser Berufsgruppe.

Aber Vorsicht! Es ist analytisch kurzschlüssig zu meinen, die Ursache der Finanzkrise ließe sich maßgeblich auf den giergetriebenen, moralischen Verfall von Bankern zurückführen. Eine solche moralische Ethisierung der Krise übersieht die politisch und gesetzlich geschaffenen Freiräume für die Finanzwirtschaft, ohne die sie ihre destruktiven Kräfte nicht hätte entfalten können. Eine solche Ethisierung übersieht also, dass die Krise - wenn auch politisch nicht beabsichtigt - sehr wohl 


\section{Warum Prävention ein Euphemismus ist}

bewusste politische Entscheidungen voraussetzt. Ohne politische Wegbereitung hätte die Krise so nicht erfolgen können. Eine politische Finanzkompetenz ist gegenwärtig auf mindestens drei Ebenen erforderlich. Sie betrifft erstens die fatale Entwicklung wachsender privater Armut, insbesondere auch der Altersarmut. Sie betrifft zweitens den Bereich des Substanzverlustes der öffentlichen Daseinsvorsorge in den Kommunen und in den Ländern, also den Bereich der öffentlichen Armut und sie betrifft drittens die nationale und internationale Schulden- und Finanzkrise. Alle drei sind nicht voneinander isolierbar, bedürfen aber dennoch der gesonderten Betrachtung.

\section{Armut in Arbeit heißt auch Armut im Alter}

In Deutschland arbeitet gegenwärtig knapp ein Viertel aller Beschäftigten im Niedriglohnbereich, also zu einem Entgelt von unter zwei Dritteln des mittleren Stundelohns. 2014 lag der Niedriglohnschwellenwert bei 9,54 Euro (vgl. Niedriglohn bleibt, 2014). Von der Einführung des gesetzlichen Mindestlohns von 8,50 Euro profitieren zwar rund 6,5 Millionen Beschäftigte, deren Stundelohn teilweise bei unter 7 Euro lag, er ändert aber nichts an der Tatsache, dass diese Menschen überwiegend keine Verbesserung ihrer sozialen Situation erfahren. Denn letztlich bedeutet die Einführung des Mindestlohns lediglich, dass der Aufwand der ergänzenden Transferleistung (Aufstockung) - überwiegend in Form von Kosten der Unterkunft nach dem $S G B I I$ - sinkt, die reale Einkommenssituation der Betroffenen aber keinerlei Verbesserung erfährt (vgl. Der Mindestlohn wirkt, 2015). Der Niedriglohnsektor hat sich rapide vom Ende der 90er Jahre bis zum Jahr 2006 entwickelt und verharrt seitdem auf einem gleichbleibenden Niveau (Brenke et al., 2011, S. 9). Dass diese Entwicklung durchaus politisch gewollt und präpariert wurde, zeigt nicht nur der politisch geförderte Ausbau der geringfügigen Beschäftigung durch die rot-grüne Regierung, sondern er ist auch grundsätzlich staatstragend angekündigt in dem sogenannten Schröder-Blair-Papier vom 8. Juni 1999. Dort heißt es: „Beschäftigungshindernisse in Sektoren mit relativ niedriger Produktivität müssen verringert werden, wenn Arbeitnehmer, die von dem mit jedem Strukturwandel einhergehenden Produktivitätszuwächsen verdrängt wurden, anderswo Arbeit finden sollen. Der Arbeitsmarkt braucht einen Sektor mit niedrigen Löhnen, um gering Qualifizierten Arbeitsplätze verfügbar zu machen“ (Der Weg nach vorne, 1999, S. 12). Obwohl der Niedriglohnsektor nicht weiter angestiegen ist, verzeichnen wir einen Rückgang der durchschnittlichen Einkommen auch über das Jahr 2005 hinaus. Laut DIW beträgt der durchschnittliche Kaufkraftverlust zwischen 2000 und 2010 vier Prozent, fast 20 Prozent in den unteren Einkommensdezilen, in den oberen ein leichtes Plus von zwei Prozent (Brenke et al., 2011, S. 11f.). Über 2,6 Millionen Beschäftigte stocken ihr Verdienst durch einen Zweitjob auf (Wenn der Job nicht reicht, 2013, S. 17). Das wachsende Phänomen 


\section{Warum Prävention ein Euphemismus ist}

des working-poor ist auch durch die steigende Zahl derjenigen indiziert, die trotz Vollzeitbeschäftigung ergänzende Hartz-IV-Leistungen benötigen, ihre Zahl lag bereits 2012 bei 350 Tausend und ist seitdem relativ konstant (Deutscher Gewerkschaftsbund, 2012, S. 2). Dies sind nur die offiziellen Zahlen der effektiven Beanspruchung von Transferleistungen. Zu erinnern ist eine Simulationsstudie des Instituts für Arbeitsmarkt- und Berufsforschung (IAB) von 2013, nach der zwischen 3,1 bis 4,9 Millionen Menschen Anspruch auf Hartz-IV-Leistungen hätten, diese aber aus Unwissenheit oder Scham nicht beantragen (vgl. Bruckmeier et al., 2013). Man könnte auch sagen: Hier spiegelt sich auch eine Finanzkompetenz der Betroffenen, lieber mit weniger Geld zu haushalten, als sich den schweren Gang zur Jobbörse aufzubürden.

Die Einkommensentwicklung in Deutschland macht unmissverständlich deutlich, dass Erwerbsarbeit für viele nicht gleichbedeutend ist mit der armutsfesten Sicherung des Lebensunterhalts. Dies betrifft aber vor allen Dingen auch die Perspektive im Alter. Drei Effekte wirken hier zusammen: Erstens wurde die Lebensarbeitszeit verlängert. Der Rentenbezug greift sukzessive erst mit 67, die Abschläge werden entsprechend hoch sein, zumal viele von denen, die bis 67 arbeiten, wegen zwischenzeitlicher Arbeitslosigkeit oder zu spätem Eintritt ins Berufsleben keine 45 Jahre sozialversicherungspflichtige Beschäftigungszeit nachweisen können. Zweitens wird der Rentensatz sukzessive von 59 Prozent in 2003 auf 43 Prozent im Jahr 2030 abgesenkt, ein finanzieller Einbruch, der selbst durch eine kapitalgedeckte Alterssicherung etwa durch die Riesterrente nicht aufgefangen werden kann, so weit diese überhaupt finanziell machbar ist (Das Ende des Riester-Booms, 2013, S. 17). Schließlich spielt sich das Ganze im Rahmen einer im Durchschnitt Kaufkraft einbüßenden Lohnentwicklung ab. Es verwundert daher nicht, dass die Neuzugangsrenten gegenwärtig durchschnittlich auf dem Niveau der frühen 1990er Jahre liegen, also inflationsbereinigt um 20 Prozent nach unten gehen (Joebges et al., 2012, S. 12). Mit anderen Worten: Eine Verschärfung der Altersarmut ist eindeutig zu prognostizieren.

Warum das alles funktioniert, ohne in Deutschland zu einer Revolte zu führen, ist durchsichtig. Schlimmer geht immer, das heißt die Widerstandslosigkeit, mit der die lohnabhängige Bevölkerung in Deutschland das sinkende Lohnniveau und die wachsende Altersarmut, working poor und prekäre Arbeitsverhältnisse klaglos in Kauf nimmt, ist dem Repressionsapparat der Hartz-IV-Maschinerie geschuldet. Denn bevor man diesem öffentlich als „Schmarotzer“ diffamierten Personenkreis angehört, nimmt man lieber alles andere in Kauf, Hauptsache Arbeit. Das ist eine Sache der Ehre, auch wenn diese Ehre ein Leben in verschämter Armut zur Folge hat. 
Warum Prävention ein Euphemismus ist

\section{Leistungskürzung ist politische Programmatik}

Die Absenkung der Rentenleistung, die Auflösung der paritätischen Krankenversicherungsfinanzierung durch Deckelung des Arbeitsgeberanteils auf 7,3 Prozent, die Kürzung der Ausgaben und der Hilfen für Menschen in Arbeitslosigkeit (Bundesrechnungshof, 2012, S. 28), die Aufforderung zur Übernahme von Eigenverantwortung in Form der Pflegeversicherung und der Riesterrente - diese Maßnahmen sind einer eindeutigen politischen Programmatik geschuldet. Man muss geradezu respektvoll zugestehen, dass kaum eine politisch-programmatische Ankündigung mit der Konsequenz umgesetzt worden ist, wie die am 14. März 2003 vom damaligen Bundeskanzler Gerhard Schröder verkündete Agenda 2010. Einige Zitate daraus: „Wir werden Leistungen des Staates kürzen, Eigenverantwortung fördern und mehr Eigenleistung von jedem Einzelnen abfordern müssen. Alle Kräfte werden ihren Beitrag leisten müssen: Unternehmer und Arbeitnehmer, freiberuflich Tätige und auch Rentner“" (Regierungserklärung, 2003). Zwar kündigt er quasi mit dem Gestus der Fürsorglichkeit an, dass die Lohnnebenkosten eine Höhe erreicht hätten, „die für Arbeitnehmer zu einer kaum mehr tragbaren Belastung geworden sind“ (ebd.), aber die komplette Logik bleibt unerwähnt: Senkung der Lohnnebenkosten bedeutet natürlich zwangsweise auch Kürzung der Leistungen im Versicherungsfall, es sei denn, es wird durch Übernahme von teuerer Eigenverantwortung ohne paritätische Finanzierung zugezahlt. Das Ganze ist eine gewaltige interne Umverteilungsmaschinerie zu Lasten der Beschäftigten und zu Gunsten der internationalen Wettbewerbsfähigkeit durch Stärkung der Exportkraft, denn die Kürzung des Arbeitgeberanteils senkt die Lohnkosten und deren Anteil an den Gütern, also die sogenannten Lohnstückkosten, übrigens auch zu Lasten anderer europäischer Länder, die die Löhne deutlich erhöht haben (vgl. Horn, 2011).

\section{Wohnst du schon oder lebst du noch - Schreckensnachrichten vom Mietmarkt}

Diese skizzierten Aspekte der Entwicklung privater, lohnbedingter Armut werden nun noch verschärft durch zwei wesentliche Aspekte: Zum einen durch einen erheblichen Anstieg der Mieten, insbesondere in den urbanen Zuzugszentren der Republik, inklusive übrigens der steigenden energetischen Mietnebenkosten. Zum anderen durch die gravierende Ver- und Überschuldung der Kommunen und auch der Länder, deren Auswirkung sich im Zuge der Verpflichtungen des sogenannten Fiskalpaktes noch massiv verschärfen wird. Zunächst zu den Mieten. Eine Bertelsmann Studie unter dem Titel „Wohnungsangebote für arme Familien in Großstädten“ belegt, dass Familien, die weniger als 60 Prozent des ortsüblichen mittleren Einkommens verdienen in 60 der 100 größten deutschen Städte nach Abzug der Miete im Durchschnitt 


\section{Warum Prävention ein Euphemismus ist}

weniger Geld zur Verfügung haben als eine Familie in Hartz-IV-Bezug (vgl. Wohnungsangebote, 2013). So verbleiben beispielsweise in Jena einer solchen „Familie mit zwei Kindern nach Überweisung der Miete rechnerisch nur 666 Euro pro Monat. Das verfügbare Einkommen liegt demnach 43 Prozent unter der staatlichen Grundsicherung, auf die eine vergleichbare Familie ohne Erwerbseinkommen Anspruch hat und die bundesweit einheitlich 1169 Euro beträgt" (Pressemeldung Bertelsmann Stiftung, 2013, S. 2). Ähnliche Auswirkungen haben hohe Wohnkosten in Frankfurt/Main, Freiburg oder Regensburg. In Heilbronn, Iserlohn, Witten oder BergischGladbach ist der Trend gegenläufig, hier liegt das verbleibende Einkommen mindestens 45 Prozent über der staatlichen Grundsicherung. Dies ist nur ein Indiz dafür, dass von einer Vergleichbarkeit der örtlichen Lebensbedingungen keine Rede sein kann und viele urbane Zuzugsräume mietbedingte Brutstätten verdeckter Armut sind.

\section{In Sonneberg wird es halbdunkel}

Was nun die Verschuldung der Städte anbelangt, so sind die Meldungen dazu alarmierend. "Griechenland ist überall in Deutschland", so kommentierte die Süddeutsche Zeitung eine Umfrage der Wirtschaftsprüfer-Gesellschaft EY (Ernst and Young) (Deutschland, wenn es dunkel wird, 2013. S. 2; Kommunen in der Finanzkrise, 2013). Erst wenige Tage zuvor war die Presse mit dem Kommunalen Finanzreport der Bertelsmann-Stiftung befasst (vgl. Kommunaler Finanzreport, 2013). Diese Studie hatte bilanziert, dass die Gesamtschulden der Kommunen 306 Milliarden Euro betragen, allein in NRW sind es 47 Milliarden. Dazu erklärte einer der Mitverfasser, René Geißler: „Die Spaltung in arme und reiche Kommunen vertieft sich“, viele Städte steckten ,in einer Abwärtsspirale aus Überschuldung, Abwanderung und sinkender Attraktivität“" (NRW-Städte überziehen, 2013, S. 1). Die Sparreaktion der Kommunen nimmt zum Teil skurrile Formen an. „Laut EY geizen etwa 14 Prozent aller Kommunen an der Straßenbeleuchtung oder wollen es in Zukunft tun“. In Sonneberg, in Thüringen bleibt ,jede zweite Lampe dunkel. Andere Gemeinden dimmen... in drei Wohngebieten im thüringischen Saalfeld bleiben die Lichter zwischen 22.30 und fünf Uhr sogar aus. Wer Beleuchtung braucht, kann die Lampe per Telefonanruf für eine Viertelstunde anknipsen lassen“" (Deutschland, wenn es dunkel wird, 2013, S. 2). 16 Prozent der Kommunen wollen bei der Jugend- oder Seniorenarbeit streichen, 13 Prozent erwägen die Hallen- oder Freibäder zu schließen, fünf Prozent wollen weniger Busse oder Bahnen im öffentlichen Nahverkehr einsetzen, vier Prozent erwägen Bibliotheken oder andere kulturelle Einrichtungen dicht zu machen.

Diese und weitere Maßnahmen betreffen zentral die Lebensqualität vor Ort. Sie entscheiden über die niederschwellige Möglichkeit zur Teilnahme an Kultur, Sport und sozialer Kontaktfindung und über die Befähigung zu bezahlbarer Mobilität. Die Verarmung des öffentlichen Raums trifft ganz entscheidend diejenigen, die 


\section{Warum Prävention ein Euphemismus ist}

aufgrund ihrer privaten Armut eh schon von vielen Aspekten der gesellschaftlichen Teilhabe ausgeschlossen sind. Sie sind die doppelten Verlierer dieser Entwicklung. Zur Vollständigkeit sei nur doch darauf hingewiesen, dass diese Verschuldungssituation nicht etwa das Ergebnis übergroßer Investitionen ist. Im Gegenteil der Investitionsstau, ja Substanzverzehr der öffentlichen Güter, also der Verwaltungsgebäude, der Straßen, Brücken, des Schienennetzwerke, der Schulen und kommunalen Sozialeinrichtungen, der Freizeitparks und Spielplätze ist horrend. Die staatliche Förderbank $K f W$ beziffert den diesbezüglichen Investitionsstau inzwischen auf 100 Milliarden Euro (vgl. Investitionsstau, 2013). Allein die ausbleibende Instandsetzung von Straßen, Schienen und Wasserwegen lässt dieses Investitionsdefizit um jährlich 7,2 Milliarden Euro wachsen (vgl. Autobahn, Brücken, Bahngleise, 2013).

\section{Koste es was es wolle - wie Staat und Banken zusammenhalten}

Die Entwicklung der internationalen Finanz- und Staatsschuldenkrise kann hier nur andeutungsweise skizziert werden. Aber wenigstens einige staatliche Förderungsaspekte der Misere sind doch anzuführen. Bekanntlich hat die Lehman-BrotherPleite ihren Ausgangspunkt in der amerikanischen Immobilienblase genommen. Die Verheißungen staatlich geförderter Sozialleistungen hat sich in den USA seit Präsident Hoover am Leitbild der „Homeowner Society“ orientiert. So erklärte der Präsident George W. Bush 2004 auf dem republikanischen Parteitag zum Auftakt seiner Widerwahlkampagne: „Dank unserer Politik befindet sich das Hauseigentum in Amerika auf einem Allzeithoch. Nun setze ich ein neues Ziel: Wir wollen sieben Millionen mehr bezahlbare Häuser in den nächsten zehn Jahren schaffen. Wieder sollen mehr amerikanische Familien beim Öffnen ihrer Haustür sagen können: Willkommen in unserem Zuhause" (Steingart, 2013, S. 174).

Der Wirtschaftspublizist und Herausgeber des Handelsblatts, Gabor Steingart zeichnet in seinem Buch „Unser Wohlstand und seine Feinde“ sehr anschaulich nach, welche Konsequenzen sich aus dieser Politik ergeben haben. Die Regierung öffnete nun „die letzten Schleusen der Kreditvergabe. Mit dem American Dream Downpayment Act wurde Einkäufern aus sozial schwachen Schichten ein bisher unvorstellbares Maß an Hilfeleistung seitens des Staates garantiert" (ebd.). Die Dokumentationspflichten für die sogenannten Subprime-Kunden, also die wenig abgesicherten Kreditnehmer, wurden drastisch reduziert. Die durch Immobilienbesitz der Banken gedeckten Wertpapier, die sogenannten Mortgage-backed Securities waren in den Banken nur Durchlaufposten, wurden Sie doch von der halbstaatlichen Immobiliengesellschaft Freddie Mac und Fannie Mae aufgekauft und verschwanden damit aus den Büchern der Banken, um neue Kreditgeschäfte zu 


\section{Warum Prävention ein Euphemismus ist}

ermöglichen. Die Haftung für Fannie und Freddie übernahm der Staat. Diese Subprime-Kredite stiegen innerhalb von knapp zehn Jahren bis 2008 von weniger als eine Billionen Dollar auf knapp zehn Billionen Dollar an (ebd., S. 177). Die Rendite dieser Wertpapiere war hoch und sie wurden in Deutschland mit einem TOPRating der Güteklasse A begehrlich gehandelt. Als dann die Immobilienpreise fielen, eine Zwangsversteigerung die andere jagte, die Kreditnehmer insolvent wurden, ging die Lawine los: Erst die Liquiditätskrise der Investmentbank Bear Stearns, dann die Krise von Freddie Mac und Fannie Mae und schließlich die Konkursanmeldung der zehntgrößten US-Bank, Lehman-Brothers am 15. September 2008. Steingart resümiert: „Subprime ist die erste Krise, die in Kollaboration von Politikern, Notenbanken und Investmentbanken vorbereitet worden ist" (ebd., S. 182).

Mit anderen Worten: Die Banker allein hätten dies nicht bewerkstelligen können, wenn nicht gesetzlich die Kreditauflagen massiv gelockert, die Eigenkapitalquote massiv gesenkt und die staatliche Risikoabsicherung nicht erfolgt wäre. Hier liegt nun ein eindeutiger Beleg dafür vor, dass Menschen mit wenig finanzieller Substanz vonseiten der Banken und des Staates abenteuerliche und unseriöse Finanzierungsangebote gemacht worden sind. Es waren Kreditgeber im politischen Schulterschluss, die alle Kriterien eines finanzkompetenten und präventiven Handelns über Bord geworfen haben. Nun sollte man nicht meinen, dass dies ein rein amerikanisches Verhalten ist. Der Finanzwirtschaft mehr Freiheit zu geben, war sowohl unter Rot-Grün als auch unter Rot-Schwarz en vogue. Bereits Ende 2001 wurde von der rot-grünen Bundesregierung mit dem vierten Finanzmarktförderungsgesetz der Hedgefonds-Industrie die Tür geöffnet. Anforderungen für den Börsenhandel wurden gelockert, die Anlagemöglichkeiten von Fonds erweitert und der Derivatenhandel auch im Immobiliengeschäft erlaubt (Koalitionsausschuss, 2009, S. 2). Im März 2003 brachten SPD und Grüne ihren Antrag „Finanzplatz Deutschland weiter fördern“ ein, unterschrieben von Franz Müntefering. Darin heißt es: Es sei „darauf zu achten, dass unnötige Belastungen für die Unternehmen der Finanzdienstleistungsindustrie vermieden werden" (ebd.). Und der damalige Finanzminister Hans Eichel erklärte gegen die Bedenkenträger: Man wolle es den Anlegern ermöglichen, „von den höheren Renditen der Hedgefonds zu profitieren“ (ebd.). Unter Rot-Schwarz gab es in Sachen Finanzmarktpolitik Kontinuität. Im Koalitionsvertrag unter Federführung von SPD-Finanzminister Steinbrück war die Rede von „Produktinnovationen und neuen Vertriebswegen“, von „Bürokratieabbau“ bei der Finanzmarktregulierung, vom „Ausbau des Verbriefungsmarktes“ und von einer „Aufsicht mit Augenmaß“. Nochmals Steingart: „Für die Banken entstanden paradiesische Verhältnisse, weil man ihnen nach und nach gestattete, die kostspielige Risikovorsorge einzustellen. Das Verhältnis von Eigenkapital und verliehenem Kapital betrug vor 100 Jahren noch 40:60 Prozent und schrumpfte im Jahr 2007 auf ein Verhältnis von 5:95 Prozent“ (Steingart, 2013, S. 146). Die Ban- 


\section{Warum Prävention ein Euphemismus ist}

kenkrise erschütterte auch die europäischen Bankenhäuser. In Deutschland gingen Hypo Real Estate, Commerzbank und Areal Bank komplett oder teilweise in Staatshand über. Unter dem Label der Systemrelevanz wurden die Banken gestützt, faule Kredite übernommen, die Staatsverschuldung weiter nach oben getrieben. Sie betrug 1980239 Milliarden Euro, in der ersten Dekade des Jahrtausends stieg sie auf 800 Milliarden, Ende 2013 belief sie sich auf gut zwei Billionen Euro. Man darf fragen wie eigentlich die Schuldenbremse des Finanzpakts wie sie im August 2009 in Kraft getreten ist, perspektivisch realisiert werden soll. Die Nettokreditaufnahme des Bundes darf ab 2016 nur noch maximal 0,35 Prozent des Bruttoinlandsprodukts betragen, den Ländern ist ab 2020 jegliche Nettokreditaufnahme verboten. Wo wird zu sparen sein? Man wird zu befürchten haben, dass es die trifft, die eh schon von Armut und Rückgang der Sozialleistungen betroffen sind. Das ist eine Art Prävention der Staatsüberschuldung zu Lasten der Ärmsten und deren Armutsprävention, also eine Art Präventionskollision. Das lehrt ja gerade das Beispiel der südeuropäischen Staaten, Griechenland, Portugal und Spanien, sie sind uns historisch voraus. Die Banken haben hier mit der Attitüde der Großzügigkeit teilweise über 18 Prozent Zinsen für die Staatsanleihen abverlangt (vgl. EuroKrise, 2011). Die Sozialleistungen, die Renten, die Krankenversorgung erlebt massivste Einbrüche. Die Jugendarbeitslosigkeit ist in Griechenland und Spanien auf über 50, teilweise gar auf über 70 Prozent gestiegen.

\section{Prävention heißt radikal umsteuern}

Und was heißt jetzt Prävention? Wie erwähnt, die Frage kommt eigentlich zu spät. Der politische Wille, hier das Ruder wirklich umzureißen, ist nicht erkennbar. Natürlich wäre eine, wenn auch moderate Vermögenssteuer unverzichtbar, denn allein die Besteuerung mit einem Prozent über einem Vermögen von 2 Millionen würden dem Staat 8,9 Milliarden Euro jährlich einbringen (vgl. Bach \& Beznoska, 2012). Natürlich müsste der Spitzensteuersatz ebenso angehoben werden wie die Körperschaftssteuer, die beide unter Rot-Grün erheblich gesenkt wurden. Natürlich brauchten wir unverzichtbar eine spürbare Transaktionssteuer für den Finanzmarkt. Man darf vielleicht nur erwähnen, dass die Bilanzsumme der Deutschen Bank real zwischen 1990 und 2010 um 640 Prozent von 204 Milliarden auf knapp zwei Billionen gestiegen ist. Das alles wäre zu diskutieren, das alles wären Maßnahmen zur Prävention, das hätte Wirkung, aber hätte, hätte...

Was bleibt, ist also noch die Jobbörse in Pinneberg mit ihrem fulminanten Vorschlag: „Gehen Sie nie hungrig einkaufen. Denn dann landen meist mehr Lebensmittel in Ihrem Einkaufswagen, als Sie zeitnah verbrauchen können“! 


\section{Warum Prävention ein Euphemismus ist}

\section{Literatur}

Bach, S. \& Beznoska, M. (2012). Vermögenssteuer: Erhebliches Aufkommenspotential trotz erwartbarer Ausweichreaktionen, DIW Wochenbericht 42/2012.

Bertelsmann Stiftung. (Hrsg.) (2013). Kommunaler Finanzreport 2013. Einnahmen, Ausgaben und Verschuldung im Ländervergleich. Gütersloh: Bertelsmann Stiftung.

Brenke, K. \& Grabka, M.M. (2011). Schwache Lohnentwicklung im letzten Jahrzehnt, DIW-Wochenbericht 45/2011.

Bruckmeier, K., Pauser, J., Walwei, U. \& Wiemers, J. (2013). Simulationsrechnung zum Ausmaß der Nicht-Beanspruchung von Leistungen der Grundsicherung, IAB-Forschungsbericht 5/2013.

Bundesrechnungshof. (2012). Mitteilung an die Bundesagentur für Arbeit über die Prüfung der Steuerung der Zielerreichung in den strategischen Geschäftsfeldern I und $V a$, Bonn.

Deutscher Gewerkschaftsbund. (2012). Arm trotz Arbeit: Aufstocker sind wesentlicher Teil des Hartz IV-Systems, Arbeitmarkt auf den Punkt gebracht 03/2012.

Horn, G.A. (2011). Des Reichtums fette Beute. Wie die Ungleichheit unser Land ruiniert. Frankfurt am Main: Campus.

Jobcenter Kreis Pinneberg. (Hrsg.) (2013). Arbeitslosengeld II Ratgeber.

Joebges, H., Meinhardt, V., Rietzler, K. \& Zwiener, R. (2012). Auf dem Weg in die Alterarmut. Bilanz der Einführung der kapitalgedeckten Riester-Rente, IMK Report 73, September 2012.

Kalina, T. \& Weinkopf, C. (2012). Niedriglohnbeschäftigung 2010: Fast jede/r Vierte arbeitet für Niedriglohn, IAQ-Report. Aktuelle Forschungsergebnisse aus dem Institut Arbeit und Qualifikation 01/2012.

Kommunen in der Finanzkrise. (2013). Kommunen in der Finanzkrise. Status Quo und Handlungsoptionen, EY Kommunenstudie 2013. Ergebnisse einer Befragung von 300 deutschen Kommunen.

Steingart, G. (2013). Unser Wohlstand und seine Feinde. München: Knaus.

Wohnungsangebote für arme Familien in Großstädten. Eine bundesweite Analyse am Beispiel der 100 einwohnerstärksten Städte. (2013). Eine Studie von Timo Heyn, Dr. Reiner Braun und Jan Grade (empirica AG) im Auftrag der Bertelsmann Stiftung.

\section{Zeitungsartikel}

Autobahn, Brücken, Bahngleise. Deutschland kaputt, Süddeutsche Zeitung, 28.07.2013, Ressort Auto \& Mobil.

Das Ende des Riester-Booms, Süddeutsche Zeitung, 15.07.2013, S. 17.

Der Niedriglohn wirkt - ein bisschen, Süddeutsche Zeitung, 22.06.2015, S. 15. 


\section{Warum Prävention ein Euphemismus ist}

Deutschland, wenn es dunkel wird, Süddeutsche Zeitung, 22.08.2013, S. 2.

„, Gehen Sie nie hungrig einkaufen “, Süddeutsche Zeitung, 19.7.2013, S. 15.

NRW-Städte überziehen ihre Konten, Kölner Stadtanzeiger, 21.08.2013, S. 1.

Wenn der Job nicht reicht, Süddeutsche Zeitung, 12.08.2013, S. 17.

\section{Internetquellen}

Armut nicht nur eine Frage von Hartz IV, Pressemeldung Bertelsmann Stiftung.

[www.bertelsmann-stiftung.de/

$\left.\mathrm{cps} / \mathrm{rde} / \mathrm{xchg} / \mathrm{bst} / \mathrm{hs} . \mathrm{xsl} / \mathrm{nachrichten} \_117419 . \mathrm{htm}\right]$.

Der Weg nach vorne für Europas Sozialdemokraten. Ein Vorschlag von Gerhard Schröder und Tony Blair (London, 8. Juni 1999).

[www.glasnost.de/pol/schroederblair.html].

Euro-Krise. Zinsen für griechische Staatsanleihen auf Rekordhoch.

[www.spiegel.de/wirtschaft/unternehmen/euro-krise-zinsen-fuer-griechenlandstaatsanleihen-auf-rekordhoch-a-78249.html].

Investitionsstau von 100 Milliarden Euro.

[www.spiegel.de/wirtschaft/soziales/kommunen-ohne-neue-schulden-undohne-investitionen-a-875742.html].

Koalitionsausschuss. Die verdrängten Sünden der Heuschrecken-Bändiger.

[www.spiegel.de/politik/deutschland/koalitionsausschuss-die-verdraengtensuenden-der-heuschrecken-baendiger-a-611329.html].

Niedriglohn bleibt.

[www.hintergrund.de/201404163071/soziales/reformen/niedriglohnbleibt.html].

Regierungserklärung Bundeskanzler Gerhard Schröder (SPD) „, Mut zum Frieden und zur Veränderung (Agenda 2010) " vom 14. März 2013.

[www.faz.net/aktuell/politik/regierungserklaerung-mut-zum-frieden-mut-zurveraenderung-191452.html]. 


\section{Warum Prävention ein Euphemismus ist}

\section{Verfasser}

Prof. Dr. Uwe Becker

Evangelische Fachhochschule Rheinland-Westfalen-Lippe in Bochum

Professur für Sozialethik, Diakoniewissenschaft und Verbändeforschung

Thomas-Mann-Str. 3

D-50868 Köln

E-Mail: ubecker-koeln@t-online.de

Internet: www.becker-sozialethik.de 